\title{
Aplicação prática do processo de enfermagem a uma adolescente portadora de doença crônica
}

\author{
PRACTICAL APPLICATION OF THE NURSING PROCESS IN AN \\ ADOLESCENT SUFFERING FROM A CHRONIC DISEASE \\ APLICACIÓN PRÁCTICA DEL PROCESO DE ENFERMERÍAALA \\ ADOLESCENTE PORTADORA DE UNA ENFERMEDAD CRÔNICA
}

\section{Franca Pellison ${ }^{1}$, Márcia Mitie Nagumo ${ }^{2}$, Elizabeth Sousa da Cunha ${ }^{3}$, Luciana de Lione Melo ${ }^{4}$}

\section{RESUMO}

O objetivo deste estudo de caso foi aplicar o processo de enfermagem fundamentado nos $\mathrm{Pa}$ drões Funcionais de Sáude, NANDA e NIC para uma adolescente portadora de diabetes mellitus secundário a fibrose cística e seu cuidador, visando ao cuidado integral. As intervenções de enfermagem foram fundamentadas nos seguintes diagnósticos de enfermagem: enfrentamento ineficaz e síndrome de estresse por mudança. A utilização desses referenciais possibilitou-nos o desenvolvimento de intervenções de enfermagem efetivas proporcionando um cuidado individualizado, além de promover uma linguagem de enfermagem padronizada.

\section{DESCRITORES}

Fibrose cística.

Diabetes mellitus.

Processos de enfermagem.

Diagnóstico de enfermagem.

Adolescente.

\section{ABSTRACT}

The purpose of this case study was to apply nursing diagnoses and interventions based on the North American Nursing Diagnosis Association (NANDA) Taxonomy of Nursing Diagnoses and the Nursing Interventions Classification (NIC) on an adolescent with secondary mellitus to cystic fibrosis and on her caretaker in order to provide them full care and guidelines. The nursing interventions were based on the following diagnoses: ineffective confrontation and stress syndrome due to alterations. These references helped us develop effective nursing interventions that provided a framework for individualized care, in addition to promoting a standardized nursing language.

\section{KEY WORDS}

Cystic fibrosis.

Diabetes mellitus.

Nursing process.

Nursing diagnosis.

Adolescent.

\section{RESUMEN}

El objetivo del presente trabajo fue aplicar el proceso de enfermería fundamentados en la Taxonomia de los Diagnósticos de Enfermería de la North American Nursing Diagnosis Association NANDA y en la clasificación de las intervenciones de la Enfermería - NIC respectivamente, para una joven portadora de diabetes mellitus secundaria a la fibrosis quística y su cuidador, visando el atención integral. Las intervenciones de enfermería fueron fundamentadas en los siguientes diagnósticos: enfrentamiento ineficaz y síndrome de estrés por cambio. La utilización de esos referenciales nos ha posibilitado el desarrollo de las intervenciones de enfermería efectivas proporcionando el cuidado individual, además de promover un lenguaje estándar de enfermería.

\section{DESCRIPTORES}

Fibrosis quística.

Diabetes mellitus.

Procesos de enfermería.

Diagnósticos de enfermería.

Adolescente.
1 Graduanda do $8^{\circ}$ semestre do Curso de Enfermagem, Departamento de Enfermagem, Faculdade de Ciências Médicas UNICAMP.

2 Graduanda do $8^{\circ}$ semestre do Curso de Enfermagem, Departamento de Enfermagem, Faculdade de Ciências Médicas UNICAMP. Campinas, SP, Brasil.

3 Graduanda do $8^{\circ}$ semestre do Curso de Enfermagem, Departamento de Enfermagem, Faculdade de Ciências Médicas UNICAMP.Campinas, SP, Brasil.

4 Enfermeira. Profes sora Doutora do Departamento de Enfermagem, Faculdade de Ciências Médicas - UNICAMP Campinas, SP, Brasil. lulione@fcm.unicamp.br 


\section{INTRODUÇÃO}

O processo de enfermagem que consiste em cinco etapas inter-relacionadas - investigação, diagnóstico, planejamento, implementação e avaliação - é um método, uma forma sistemática e dinâmica de prestar os cuidados de enfermagem. Através deste método, o enfermeiro promove cuidado humanizado, dirigido a resultados e de baixo custo e, ainda, esse é capaz de impulsionar os enfermeiros a continuamente examinarem suas práticas, refletindo sobre formas de fazê-las melhor ${ }^{(1)}$.

O foco do processo de enfermagem é o indivíduo como um todo e não apenas sua doença, o que possibilita uma abordagem ampla, buscando maximizar a autonomia do indivíduo, considerando suas respostas humanas a um determinado problema, além de facilitar a interação enfermeirocliente.

A partir de 1998, a aprovação da Lei do Exercício Profissional de Enfermagem estabeleceu como atribuição privativa do enfermeiro, a prescrição de enfermagem, e a partir daí, o processo de enfermagem passou a ser alvo de maior preocupação para os enfermeiros brasileiros. Na década de 80, este fato pôde ser observado na literatura nacional mediante os diversos relatos sobre experiências de implantação de metodologias de assistência nas instituições de saúde brasileiras ${ }^{(2)}$.

Corroborando com a Lei do Exercício Profissional, a Resolução COFEN 272/2002 reintera ser função exclusiva do enfermeiro realizar o processo de enfermagem, tendo como finalidade identificar situações de saúde/doença e subsidiar ações de enfermagem que possam contribuir para promoção, prevenção, recuperação e reabilitação da saúde do indivíduo, família e comunidade ${ }^{(3)}$.

A implementação do processo de enfermagem, utilizando na fase diagnóstica, a Taxonomia dos Diagnósticos de Enfermagem da North American Nursing Diagnosis Association (NANDA) ${ }^{(4)}$ iniciou-se nos Estados Unidos da América, em meados da década de $80^{(2)}$. Porém, no Brasil, sua utilização é mais recente.

Em pesquisa realizada com o objetivo de descrever como é oferecido o ensino do Processo de Enfermagem nas Escolas de Graduação em Enfermagem, do Estado de São Paulo, o referencial teórico adotado em $52 \%$ da amostra diz respeito ao modelo de Wanda de Aguiar Horta. A referida pesquisa faz menção ao processo de enfermagem utilizando a $\mathrm{NANDA}^{(4)}$ em um único estudo realizado em uma Unidade de Queimados ${ }^{(5)}$.

O Hospital Universitário da Universidade de São Paulo (HU-USP), que há mais de duas décadas fundamenta sua prática assistencial no processo de enfermagem, começou em 2001 sua trajetória na implantação do diagnóstico de enfermagem fundamentado na NANDA ${ }^{(4)}$. Esta trajetória tem mostrado que a implantação e a implementação exigem tempo e determinação dos envolvidos ${ }^{(6)}$.

Desta forma, podemos evidenciar que a utilização das classificações de enfermagem ainda é recente no Brasil, tanto no ensino de graduação como nas instituições de saúde.

A disciplina Enfermagem na Saúde da Criança e do Adolescente II, do curso de graduação em Enfermagem, da Faculdade de Ciências Médicas da Unicamp, iniciou a abordagem ao processo de enfermagem utilizando a NANDA e a Classificação das Intervenções de Enfermagem (NIC) ${ }^{(7)} \mathrm{em}$ 2005, porém no ano anterior foram realizados em atividadespráticas desta disciplina, alguns ensaios com as referidas classificações no sentido de identificar sua real viabilidade. Além destes ensaios, construímos um histórico de enfermagem fundamentado nos Padrões funcionais de Saúde ${ }^{(8)}$.

A aplicação do processo de enfermagem fundamentado nos Padrões Funcionais de Saúde ${ }^{(8)}$, NANDA ${ }^{(4)}$ e NIC $^{(7)}$ deuse a uma adolescente portadora de diabetes mellitus secundário à fibrose cística devido a existência de uma lacuna na literatura, sobre trabalhos que abordem um cuidado específico de enfermagem, consi-derando além da patologia e fase da vida do indivíduo.

Assim, diante do exposto, a aplicação do processo de enfermagem poderá possibilitar uma melhoria na qualidade da assistência, uma vez que o planejamento e a implementação das ações de enfermagem deverão se voltar as reais necessidades desse cliente.

\section{OBJETIVO}

Aplicar o processo de enfermagem fundamentado nos Padrões Funcionais de Saúde ${ }^{(8)}$, NANDA ${ }^{(4)}$ e NIC ${ }^{(7)}$ para uma adolescente portadora de diabetes mellitus secundário a fibrose cística e seu cuidador, visando o cuidado integral.

\section{MÉTODO}

Trata-se de um estudo tipo estudo de caso. O estudo de caso é considerado um dos mais relevantes tipos de pesquisa qualitativa, permitindo realizar investigações em profundidade de um indivíduo, grupo ou instituição. Apesar de não necessitar, a priori, da elaboração de hipóteses, sua complexidade aumenta à medida que o investigador adota um ou mais referenciais teóricos ${ }^{(9-10)}$.

Foi realizado no município de Campinas - SP, em uma unidade de internação pediátrica de um hospital universitário, no $2^{\circ}$ semestre de 2004, na disciplina Enfermagem na Saúde da Criança e do Adolescente II, do curso de graduação em Enfermagem, da Faculdade de Ciências Médicas da Unicamp. 
A população foi composta por uma adolescente portadora de diabettes mellitus secundário à fibrose cística e seu cuidador, que aceitaram participar do estudo. Durante todo o processo assistencial respeitamos os aspectos éticos para pesquisa envolvendo seres humanos preconizados pela Resolução 196/96 do Conselho Nacional de Saúde ${ }^{(11)}$.

Os dados foram coletados através de entrevista e exame físico, utilizando-se um instrumento fundamentado nos Padrões Funcionais de Saúde ${ }^{(8)}$ adaptado para o adolescente hospitalizado. Vale ressaltar que dos onze padrões contidos no instrumento, foi o padrão de tolerância e resposta ao estresse que evidenciou, de fato, os problemas de enfermagem que necessitavam de cuidado prioritário.

Após a análise dos dados coletados, apesar de elaborarmos 9 diagnósticos de enfermagem segundo taxonomia North American Nursing Diagnosis Association NANDA $^{(4)}$, planejamos as intervenções de enfermagem embasadas na Classificação das Intervenções de Enferma- gem - NIC(7) de acordo com os seguintes diagnósticos: enfrentamento ineficaz e síndrome de estresse por mudança (Quadros 1 e 2).

\section{DIAGNÓSTICOS DE ENFERMAGEM DA NANDA E INTERVENÇÕES DE ENFERMAGEM DA NIC PARA ADOLESCENTE PORTADORA DE DIABETES MELLITUS SECUNDÁRIO A FIBROSE CÍSTICA E SEU CUIDADOR}

Enfrentamento ineficaz relacionado a nível inadequado de enfrentamento, incerteza, crises situacionais, distúrbio no padrão de avaliação de ameaça caracterizado por verbalização de incapacidade de enfrentamento, incapacidade de satisfazer necessidades básicas, falta de comportamento direcionado a objetivos/resolução de problemas incluindo incapacidade de lidar com informações, utilização de formas de enfrentamento que impedem o comportamento adaptativo.

Quadro 1 - Enfrentamento ineficaz

\begin{tabular}{|c|c|}
\hline Intervenções de Enfermagem & Como intervir \\
\hline Melhora do enfrentamento & $\begin{array}{l}\text { Avaliar o impacto da situação de vida da adolescente sobre papéis e relacionamentos. } \\
\text { Avaliar a compreensão que a adolescente tem do processo de doença. } \\
\text { Ajudar à adolescente a identificar as informações que ela tem mais interesse em obter. } \\
\text { Oferecer à adolescente escolhas reais sobre determinados aspectos do cuidado. } \\
\text { Avaliar a capacidade decisória da adolescente. } \\
\text { Auxiliar a adolescente a esclarecer idéias errôneas. } \\
\text { Encorajar a adolescente a avaliar o próprio comportamento. }\end{array}$ \\
\hline Apoio à tomada de decisão & $\begin{array}{l}\text { Informar a adolescente sobre soluções alternativas. } \\
\text { Ajudar adolescente a identificar as vantagens e desvantagens de cada alternativa. } \\
\text { Ajudar a adolescente a explicar a decisão aos outros conforme a necessidade. }\end{array}$ \\
\hline Grupo de apoio & $\begin{array}{l}\text { Usar um grupo de apoio durante os estágios de transição para auxiliar a adolescente a } \\
\text { adaptar-se a um novo modo de vida. }\end{array}$ \\
\hline Ensino: indivíduo & $\begin{array}{l}\text { Determinar a motivação da adolescente para aprender informações específicas. } \\
\text { Reforçar comportamentos, quando adequado. } \\
\text { Corrigir interpretações erradas das informações. } \\
\text { Proporcionar tempo para que a adolescente faça perguntas e discuta preocupações. } \\
\text { Documentar o conteúdo apresentado, os materiais escritos oferecidos e a compreensão da } \\
\text { adolescente em relação à informação que indiquem aprendizagem, em registro médico } \\
\text { permanente. }\end{array}$ \\
\hline
\end{tabular}

Síndrome de estresse por mudança relacionado a perdas recentes, imprevisibilidade da experiência, saúde psicossocial prejudicada, estado de saúde diminuído caracteriza- do por mudança permanente e involuntária, sintomas físicos/doença aumentados, insegurança, pessimismo, preocupação quanto à mudança. 
Quadro 2 - Síndrome de estresse por mudança

\begin{tabular}{|c|c|}
\hline Intervenções de Enfermagem & Como intervir \\
\hline Suporte à família & $\begin{array}{l}\text { Avaliar a reação emocional da família à condição da adolescente. } \\
\text { Determinar a carga psicológica do prognóstico para a família. } \\
\text { Identificar a coerência entre as expectativas da adolescente, da família e do } \\
\text { profissional da saúde. } \\
\text { Oferecer feedback à família quanto a seu enfrentamento. } \\
\text { Ensinar os planos médicos e de enfermagem à família. } \\
\text { Oferecer à família os conhecimentos necessários quanto às opções que a auxiliarão } \\
\text { na tomada de decisões sobre os cuidados da adolescente. } \\
\text { Encorajar o processo decisório familiar no planejamento de cuidados a longo prazo } \\
\text { que afetem a estrutura e os recursos financeiros da família. } \\
\text { Confirmar a compreensão da decisão da família em relação aos cuidados pós-alta. } \\
\text { Auxiliar a família a adquirir os conhecimentos, as habilidades e o equipamento } \\
\text { necessários para manter sua decisão sobre os cuidados da adolescente. }\end{array}$ \\
\hline Aumento da segurança & $\begin{array}{l}\text { Discutir as mudanças que estão por vir, antes que ocorra. } \\
\text { Explicar todos os testes e procedimentos a adolescente/família. } \\
\text { Auxiliar a adolescente/família a identificar os fatores que aumentam a sensação de } \\
\text { segurança. }\end{array}$ \\
\hline Facilitação da auto-responsabilidade & $\begin{array}{l}\text { Conscientizar a adolescente sobre o comportamento adequado. } \\
\text { Determinar se a adolescente possui um conhecimento adequado da condição de } \\
\text { cuidados de saúde. } \\
\text { Monitorar o nível de responsabilidade que a adolescente assume. } \\
\text { Encorajar a independência, mas assistir a adolescente quando incapaz de fazer as } \\
\text { coisas. } \\
\text { Discutir as conseqüências de não lidar com as próprias responsabilidades. } \\
\text { Evitar discutir ou barganhar a respeito dos limites estabelecidos com a adolescente. }\end{array}$ \\
\hline Monitorização nutricional & $\begin{array}{l}\text { Pesar a adolescente a intervalos de no mínimo, uma vez por semana. } \\
\text { Monitorar as tendências de perda de peso. } \\
\text { Monitorar o tipo e a quantidade de exercícios usuais. } \\
\text { Monitorar a resposta emocional da adolescente quando colocado em situações que } \\
\text { envolvam alimentos e ato alimentar. } \\
\text { Monitorar as preferências e escolhas alimentares. } \\
\text { Monitorar o crescimento e o desenvolvimento } \\
\text { Monitorar o nível de energia, mal-estar, fadiga e fraqueza. } \\
\text { Monitorar a ingestão nutricional. }\end{array}$ \\
\hline
\end{tabular}

\section{CONSIDERAÇÕES FINAIS}

A utilização dos Padrões Funcionais de Saúde, NANDA e NIC possibilitou-nos oferecer um cuidado de enfermagem efetivo, uma vez que evidenciamos que os diagnósticos e as intervenções de enfermagem colaboraram para que a adolescente pudesse, durante a hospitalização e paulatinamente, compreender suas novas necessidades.

Uma das intervenções que encontramos maior resistência por parte da adolescente diz respeito a monitorização nutricional, pois antes do diagnóstico de diabetes mellitus e devido à fibrose cística, sua dieta era hipercalórica e hiperlípidica, já que em função da doença de base há uma perda excessiva de gordura. Contudo, o diabetes mellitus impulsionou a uma mudança significativa da dieta, já iniciada no período intra-hospitalar.
O estabelecimento de uma parceria, baseada em confiança mútua, entre profissional de saúde-adolescente-família foi essencial para encorajar a adolescente a assumir um papel ativo no seu plano de cuidados. Esta parceria exigiu que nós - profissionais de saúde - mudássemos da abordagem cuidarei de você para como posso fortalecê-la para torná-la independente? ${ }^{(2)}$. Foi necessário que compreendêssemos a adolescente como a melhor conhecedora de si e demonstrássemos que estávamos partilhando um mesmo objetivo.

Este modo de cuidar do outro, fez-nos refletir sobre a formação biologicista, com ênfase na patologia que vem sendo oferecida. Apesar disso, faz-se necessário extrapolar o corpo, buscando compreender o indivíduo em sua totalidade, considerando o contexto no qual está inserido. 
A dificuldade inicial de manuseio da NANDA e NIC, foi, ao poucos, sendo sanada pelo uso contínuo deste referencial apoiado no raciocínio clínico que promoveu, concomitantemente, o aprendizado de uma linguagem padronizada

\section{REFERÊNCIAS}

1. Alfaro-Lefevre R. Aplicação do processo de enfermagem: promoção do cuidado colaborativo. Porto Alegre: Artmed; 2005.

2. Rossi LA, Casagrande LDR. Processo de enfermagem: a ideologia da rotina e a utopia do cuidado individualizado. In: Cianciarullo TI, Gualda DMR, Melleiro MM, Anabuki MH, organizadoras. Sistema de Assistência de Enfermagem: evolução e tendências. São Paulo: Ícone; 2001. p. 41-62.

3. Conselho Federal de Enfermagem (COFEN). Resolução n. 272/ 2002. Dispõe sobre a Sistematização da Assistência de Enfermagem - SAE, nas Instituições de Saúde Brasileiras. Brasília; 2002.

4. North American Nursing Diagnosis Association (NANDA). Diagnósticos de enfermagem da NANDA: definições e classificação: 2003-2004. Porto Alegre: Artmed; 2005.

5. Dell’Acqua MCQ, Miyadahira AMK. Ensino do processo de enfermagem nas escolas de graduação em enfermagem do Estado de São Paulo. Rev Lat Am Enferm. 2002;10(2):185-91. de enfermagem e a evidência de um planejamento voltado às reais necessidades da adolescente, resultando na melhoria das condições de saúde.

6. Lima AFC, Kurcgant P. O processo de implementação do diagnóstico de enfermagem no Hospital Universitário da Universidade de São Paulo. Rev Esc Enferm USP. 2006;40(1):111-6.

7. Mc Closkey JC, Bulechek GM, organizadoras. Classificação das Intervenções de Enfermagem (NIC). Porto Alegre: Artmed; 2004.

8. Gordon M. Manual of nursing diagnosis. St. Louis: Mosby; 1998.

9. Polit DF, Hungler BP. Nursing research: principles and methods. Philadelphia: Lippincott; 1987.

10. Triviños ANS. Introdução a pesquisa em ciências sociais: a pesquisa qualitativa em educação. São Paulo: Atlas; 1992.

11. Conselho Nacional de Saúde. Resolução n. 196, de 10 de outubro de 1996. Dispõe sobre diretrizes e normas regulamentadoras de pesquisas envolvendo seres humanos. Bioética. 1996;4(2 Supl):15-25. 